\title{
Femtosecond time-resolved optical pump-probe spectroscopy at kilohertz-scan-rates over nanosecond-time-delays without mechanical delay line
}

\author{
A. Bartels, ${ }^{\text {a) }}$ F. Hudert, C. Janke, and T. Dekorsy \\ Department of Physics and Center for Applied Photonics, University of Konstanz, D-78457 Konstanz, \\ Germany \\ K. Köhler \\ Fraunhofer-Institut für Angewandte Festkörperphysik, D-79108 Freiburg, Germany
}

\begin{abstract}
We demonstrate a technique for femtosecond time-resolved optical pump-probe spectroscopy that allows to scan over a nanosecond time delay at a kilohertz scan rate without mechanical delay line. Two mode-locked femtosecond lasers with approximately $1 \mathrm{GHz}$ repetition rate are linked at a fixed difference frequency of $\Delta f_{R}=11 \mathrm{kHz}$. One laser delivers the pump pulses, the other provides the probe pulses. The relative time delay is linearly ramped between zero and the inverse laser repetition frequency at a rate $\Delta f_{R}$, enabling high-speed scanning over a $1 \mathrm{~ns}$ time delay. The advantages of this method for all-optical pump-probe experiments become evident in an observation of coherent acoustic phonons in a semiconductor superlattice via transient reflectivity changes. A detection shot-noise limited signal resolution of $7 \times 10^{-8}$ is obtained with a total measurement time of $250 \mathrm{~s}$. The time resolution is $230 \mathrm{fs}$.
\end{abstract}

Experiments in the field of ultrafast time-domain spectroscopy most commonly employ trains of pump and probe pulses derived from a single mode-locked laser. One pulse train travels a variable distance before arriving at the sample and data are recorded as a function of the time delay versus the other pulse train. In most cases the time delay is adjusted with mirrors mounted onto a mechanical translation stage or a vibrating membrane. Translation stages allow for several hundred picoseconds delay but not for rapid scanning. Vibrating membranes allow for higher scan rates in the range of $100 \mathrm{~Hz}$ but are limited to approximately 10 ps time delay. A severe disadvantage of moving mirrors in general is the fact that it is impossible to eliminate residual variations of the beam pointing as time delays of several hundred picoseconds are realized by moving the stage by tens of centimeters. Also, spot size variations at the sample position are inherent due to the divergence of the laser beam. These effects are greatly unfavorable when phenomena with long decay and/or dephasing times are investigated over nanosecond time delays due to an unresolvable ambiguity between real sample dynamics and experimental artifacts. Examples of physical phenomena that require the detection of sample dynamics over nanosecond time delays are picosecond ultrasound pulses, ${ }^{1-3}$ transient spin-dynamics, ${ }^{4,5}$ long-living coherent lattice or charge carrier dynamics in solid-state, ${ }^{6,7}$ highresolution terahertz spectroscopy, ${ }^{8}$ terahertz ranging, ${ }^{9}$ or the characterization of high-speed optoelectronic devices. ${ }^{10}$ Furthermore, increased scan rates are desirable for imaging applications to reduce pixel dwell times or for experiments where data have to be acquired in a short period of time (e.g., pulsed magnetic fields).

Asynchronous optical sampling (ASOPS) is a technique demonstrated earlier with mode-locked picosecond lasers at low repetition rates $(82 \mathrm{MHz})$ that enables ultrafast time-

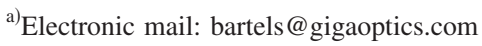

domain spectroscopy without a mechanical delay line. ${ }^{11}$ To this end, two mode-locked lasers are linked at a fixed repetition rate difference $\Delta f_{R}$, and serve as pump and probe lasers. Their relative time delay is linearly ramped between zero and the inverse repetition rate of the pump laser at a rate given by $\Delta f_{R}$. Our high-speed implementation of ASOPS operates with mode-locked Ti:sapphire femtosecond lasers at $1 \mathrm{GHz}$ repetition rate and thus allows kilohertz scan rates over a temporal measurement window of $1 \mathrm{~ns}$ with femtosecond time resolution. High repetition rates in the range of $1 \mathrm{GHz}$ are generally favorable for high-speed ASOPS because they reduce the required optical detection and data acquisition bandwidth $F$ to practically accessible values in the range of $100 \mathrm{MHz}$. The feasibility of high-speed ASOPS has recently been shown in the field of terahertz time-domain spectroscopy. ${ }^{12}$ However, the time resolution had been limited to 2.5 ps due to limitations of the laser stabilization and a restricted bandwidth of the detection system.

In this letter, we report the application of high-speed ASOPS to all-optical femtosecond time-resolved pumpprobe spectroscopy. As an important prerequisite, we have significantly improved and simplified the electronics used to stabilize the laser repetition rate difference. To demonstrate the utility of the technique, we have chosen a GaAs/AlAs semiconductor superlattice sample as a challenging model system. The response of such structures to impulsive optical excitation is well known from earlier experiments performed conventionally with mechanical delay lines: Coherent longitudinal acoustic phonons with periods on the order of $1 \mathrm{ps}$ and lifetimes exceeding $100 \mathrm{ps}$ are observable through weak transient reflectivity changes.

We employ a dual-laser system (Gigajet TWIN, Gigaoptics $\mathrm{GmbH}$, Germany) which comprises two Ti:sapphire femtosecond oscillators with repetition rates $f_{R, 1}$ (laser 1) and $f_{R, 2}$ (laser 2) of approximately $1 \mathrm{GHz}$. The oscillators are pumped by a diode-pumped solid-state laser at $532 \mathrm{~nm}$ wavelength whose $6.5 \mathrm{~W}$ output power is split into equal 

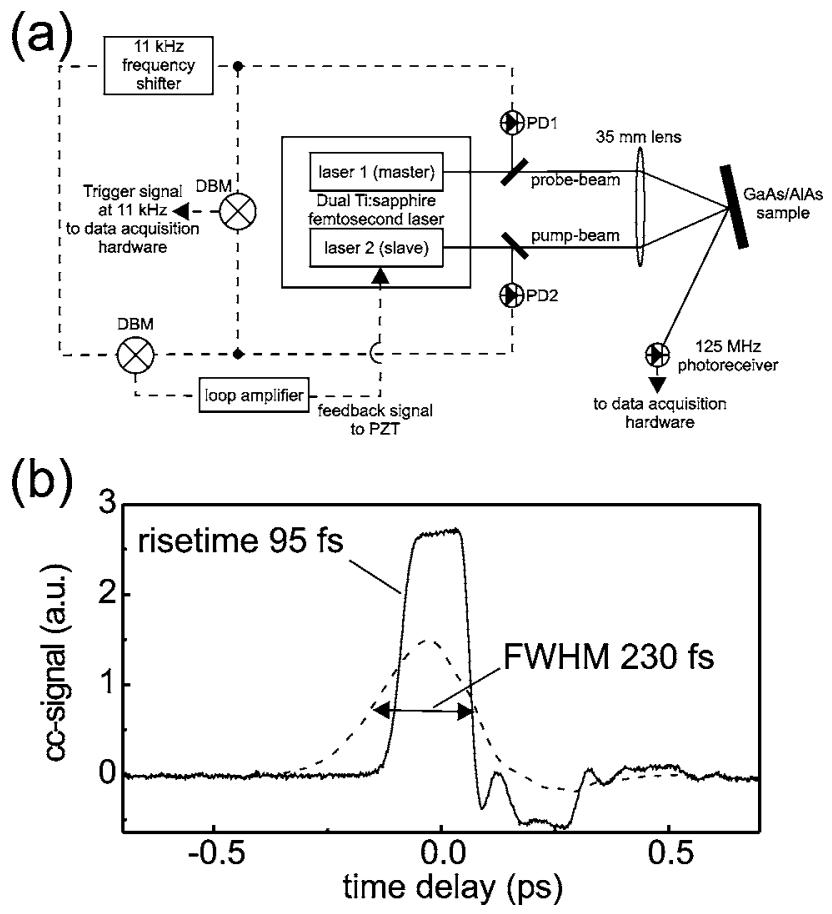

FIG. 1. (a) Experimental setup. Solid lines are optical paths, dashed lines represent electronic signals. See text for details. (b) Cross-correlation signal between lasers 1 and 2: single scan (solid line) and averaged over 512 scans (dashed line).

portions. The oscillators deliver approximately $500 \mathrm{~mW}$ of output power at a center wavelength of $820 \mathrm{~nm}$ with a pulse duration of approximately 30 fs. The repetition rate difference $\Delta f_{R}=f_{R, 1}-f_{R, 2}$ is fixed using active feedback. The time delay between the two pulse trains is then $\tau(t)=t \times \Delta f_{R} / f_{R, 2}$ modulo $f_{R, 2}^{-1}$ if laser 2 is used as the pump laser and $t$ is the real time. The quality of the feedback electronics determines the accuracy of the $\tau(t)$ relationship given above through a jitter of the time base which directly determines the experimental time resolution. Instead of employing two feedback loops to stabilize the lasers to their individual reference synthesizers with an offset of $\Delta f_{R}$, ${ }^{12}$ we use laser 1 as master laser and link laser 2 as slave laser to it at a difference of $\Delta f_{R}=11 \mathrm{kHz}$. This has the advantage that only the residual errors of one feedback loop enter the jitter of the time-base.

The experimental scheme is sketched in Fig. 1(a). We measure the $f_{R, 1}$ by detecting laser 1 with a fast photodiode (PD1) and upshift the frequency of the electronic signal by $11 \mathrm{kHz}$ using a frequency shifter. The frequency shifter consists of a single-sideband generator with a carrier suppression stage that allows a suppression of the initial carrier signal by better than $50 \mathrm{~dB}$. Subsequently, $f_{R, 2}$ is detected with a second photodiode (PD2) and phase locked to the frequencyshifted repetition rate signal of laser 1 . To accomplish this, we use a double-balanced mixer (DBM) as phase detector and feed its output back to a piezoelectric transducer that supports a cavity mirror in laser 2 via an adjustable loop amplifier. The jitter of the time-base $\tau(t)$ is characterized by detecting a cross-correlation signal between the two lasers using noncollinear sum-frequency generation in a $\beta$-barium-borate crystal. The cross-correlation signal is detected with a $125-\mathrm{MHz}$ bandwidth photoreceiver and recorded using a sampling oscilloscope (Tektronix TDS3032B). To generate a trigger signal for the oscilloscope at $\Delta f_{R}$, we use a second DBM with the signals from PD1 and
PD2 as inputs. The real-time scale is converted to a timedelay scale by applying a factor $\Delta f_{R} / f_{R, 2}$. Figure 1(b) shows a single scan measurement of the cross-correlation signal and an average over 512 measurements acquired over $\approx 1 \mathrm{~s}$, limited by the averaging function of the oscilloscope. The single scan trace with a rise time of 95 fs shows the limitation to the time-resolution $\Delta \tau$ given by the $100 \mathrm{MHz}$ bandwidth of the sampling oscilloscope (determined by our specific chosen acquisition settings) according to $\Delta \tau=\Delta f_{R} / f_{R, 2} F$, where $F$ is the effective bandwidth of the photodetection and data acquisition electronics. The detailed structure is attributed to the response of the photoreceiver. The averaged crosscorrelation signal has a width of $230 \mathrm{fs}$, reflecting residual jitter of the time base. Averaging over longer times does not further broaden the signal, thus $230 \mathrm{fs}$ represents the overall time resolution of our setup. This limit is mostly due to phase-noise intrinsic to the DBMs used for phase detection and trigger generation as well as a residual contribution at the carrier frequency at the output of the frequency shifter. We envision that an improvement of the timing stability to the sub-100 fs regime is possible by performing the phase lock at a higher harmonic of the repetition rate or by using digital phase detectors.

All-optical pump-probe spectroscopy is performed on a GaAs/AlAs semiconductor superlattice sample with 40 periods of 18 atomic monolayers of GaAs and 18 monolayers of AlAs. The pump and probe beams are focused onto the same spot on the sample using a lens with a focal length of $35 \mathrm{~mm}$. The spot size is approximately $30 \mu \mathrm{m}$. The pump and probe powers are 400 and $10 \mathrm{~mW}$, respectively. The excitation wavelength is close to the interband transition between the first quantized electron and hole states. After reflection off the sample, the probe beam is focused onto the $125-\mathrm{MHz}$ bandwidth photoreceiver; reference detection is not used. The signal is recorded with a $200 \mathrm{Ms} / \mathrm{s} 14$ Bit analog-todigital converter (ADC) on a PCI board (Compuscope 14200, GaGe Applied Technologies) that is triggered by the synchronization electronics. The data acquisition board has the capability of acquiring hardware-based averages over 1024 single scan traces. These averaged traces are displayed at a rate of $4 \mathrm{~Hz}$ on the computer. An arbitrary number of additional software based averages can be computed. The spatial overlap of pump and probe pulse can be optimzed "on-line" by observing the signal transient on our data acquisition computer. An average over $1024 \times 1000$ single traces with a total acquisition time of $250 \mathrm{~s}$ is shown in Fig. 2(a). Data are calibrated to show relative reflectivity changes $\Delta R / R_{0}$, where $R_{0}$ is the unperturbed reflectivity.

The signal exhibits a rise in reflectivity of $1.5 \times 10^{-3}$ due to optically excited charge carriers in the superlattice followed by a decay due to relaxation, recombination, and diffusion. Sufficient signal-to-noise ratio to obtain the electronic dynamics can already be achieved without software averaging. Superimposed on the electronic signature is a modulation of the reflectivity with an amplitude of $\approx 2$ $\times 10^{-6}$ due to impulsively driven coherent acoustic phonons. Their oscillatory contribution has been extracted by subtracting a fit to the slow electronic dynamics from the original data and is shown in Fig. 2(b). A fast-Fourier-transformation (FFT) of the data is shown in Fig. 2(d) (left panel), revealing two dominating modes at 41.8 and $44.5 \mathrm{GHz}$. These frequencies agree well with those of propagating unfolded longitudinal acoustic phonons driven in the individual GaAs and 

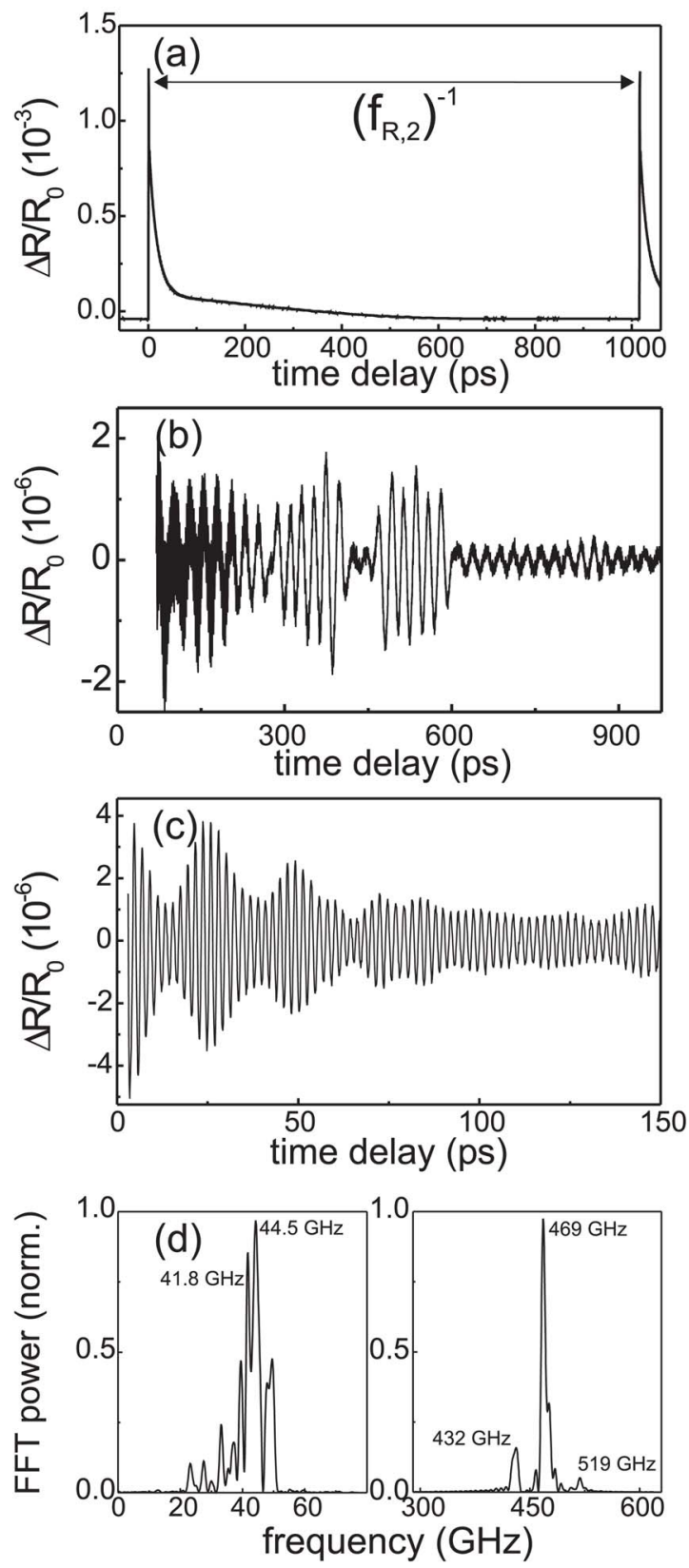

FIG. 2. (a) Transient reflectivity changes of the GaAs/AlAs superlattice sample after impulsive excitation. (b) Signature of unfolded longitudinal acoustic phonons in the sample. First 60 ps are not shown, because higher frequency phonons would smear out the data to a broad band. (c) Signature of first-order zone-folded longitudinal acoustic phonons. Only the first 150 ps are shown for better visibility. (d) FFT data of the traces shown in (b) and (c).

AlAs layers via impulsive Raman excitation in a backscattering geometry with a wave-vector $q=2 k_{820}$ that are theoretically expected around 42.2 and $45.7 \mathrm{GHz}$. Here, $k_{820}$ is the light wave-vector inside the sample. The strongly reduced amplitude after $\tau \approx 600 \mathrm{ps}$ is due to the fact that the coherent phonon burst propagates out of the superlattice and thus cannot be detected anymore. ${ }^{7}$ The signal also contains a much faster modulation due to first-order zone-folded acoustic phonons that cannot be graphically resolved on the $1 \mathrm{~ns}$ scale in Fig. 2(b). This contribution has therefore been extracted as above and is shown in Fig. 2(c). The FFT [Fig. 2(d), right panel] shows three modes that are attributed to a nonpropagating zone-folded acoustic phonon mode at $q=0(469 \mathrm{GHz})$ and two propagating zone-folded acoustic phonon modes with $q=2 k_{820}(432$ and $519 \mathrm{GHz}){ }^{6}$ A detailed statistical analysis of data acquired at different numbers of software averages $N$ shows that the signals are shot-noise limited with a noise dependence $\propto \sqrt{N}$. At $N=1000$, the resolution for $\Delta R / R_{0}$ is $7 \times 10^{-8}$. In order to acquire equivalent data with an earlier demonstrated method based on a vibrating membrane, ${ }^{6}$ a total data acquisition time of $\approx 10000$ s would have been required. It is important to note, however, that this method would not have been capable to resolve the low frequency excitations around $45 \mathrm{GHz}$.

The presented data qualify high-speed ASOPS as a technique for femtosecond time-resolved optical pump-probe spectroscopy that is comparable to conventional methods in terms of time resolution and signal-to-noise ratio and is largely superior in a number of other respects: It allows for the elimination of mechanical delay lines and thus variations of beam pointing and spot size as well as acoustic noise due to mechanical movement. Furthermore, we have demonstrated a scan rate of $11 \mathrm{kHz}$ over a measurement window of 1 ns with 230 fs time resolution and obtained a signal resolution of $7 \times 10^{-8}$ within a total sampling time of $250 \mathrm{~s}$. This is an improvement by more than one order of magnitude compared to the time required for mechanical delay scanning.

The authors thank E. N. Ivanov, S. A. Diddams, and S. Eggert for their contributions to the frequency shifter. This work has partially been supported by Gigaoptics $\mathrm{GmbH}$, Deutsche Forschungsgemeinschaft (De 567/9-1), and the Ministry of Science, Research and the Arts of BadenWürttemberg.

${ }^{1}$ C. Thomsen, H. T. Grahn, H. J. Maris, and J. Tauc, Phys. Rev. B 34, 4129 (1986).

${ }^{2}$ O. L. Muskens and J. I. Dijkhuis, Phys. Rev. B 70, 104301 (2004).

${ }^{3}$ G. D. Cahill, W. K. Ford, K. E. Goodson, G. D. Mahan, A. Majumdar, H. J. Maris, R. Merlin, and S. R. Phillpot, J. Appl. Phys. 93, 793 (2003).

${ }^{4}$ J. M. Kikkawa and D. D. Awschalom, Phys. Rev. Lett. 80, 4313 (1998); Science 287, 473 (2000).

${ }^{5}$ R. C. Myers, K. C. Ku, X. Li, M. Samarth, and D. D. Awschalom, Phys. Rev. B 72, 041302 (2005).

${ }^{6}$ A. Bartels, T. Dekorsy, H. Kurz, and K. Köhler, Phys. Rev. Lett. 82, 1044 (1999), and references therein.

${ }^{7}$ K. Mizoguchi, M. Hase, S. Nakashima, and M. Nakayama, Phys. Rev. B 60, 8262 (1999).

${ }^{8}$ S. A. Harmon and R. A. Cheville, Appl. Phys. Lett. 85, 2128 (2004).

${ }^{9}$ R. A. Cheville and D. Grischkowsky, IEEE Trans. Microwave Theory Tech. 38, 1684 (1995).

${ }^{10}$ M. Bieler, M. Spitzer, G. Hein, U. Siegner, and E. O. Göbel, Appl. Phys. A 78, 429 (2004).

${ }^{11}$ P. A. Elzinga, F. E. Lytle, Y. Jian, G. B. King, and N. M. Laurendeau, Appl. Opt. 26, 4303 (1987).

${ }^{12}$ C. Janke, M. Först, M. Nagel, H. Kurz, and A. Bartels, Opt. Lett. 30, 1405 (2005). 